\title{
«Die Work-Life-Balance ist wichtiger als der Lohn»
}

Interview: Anna Sax

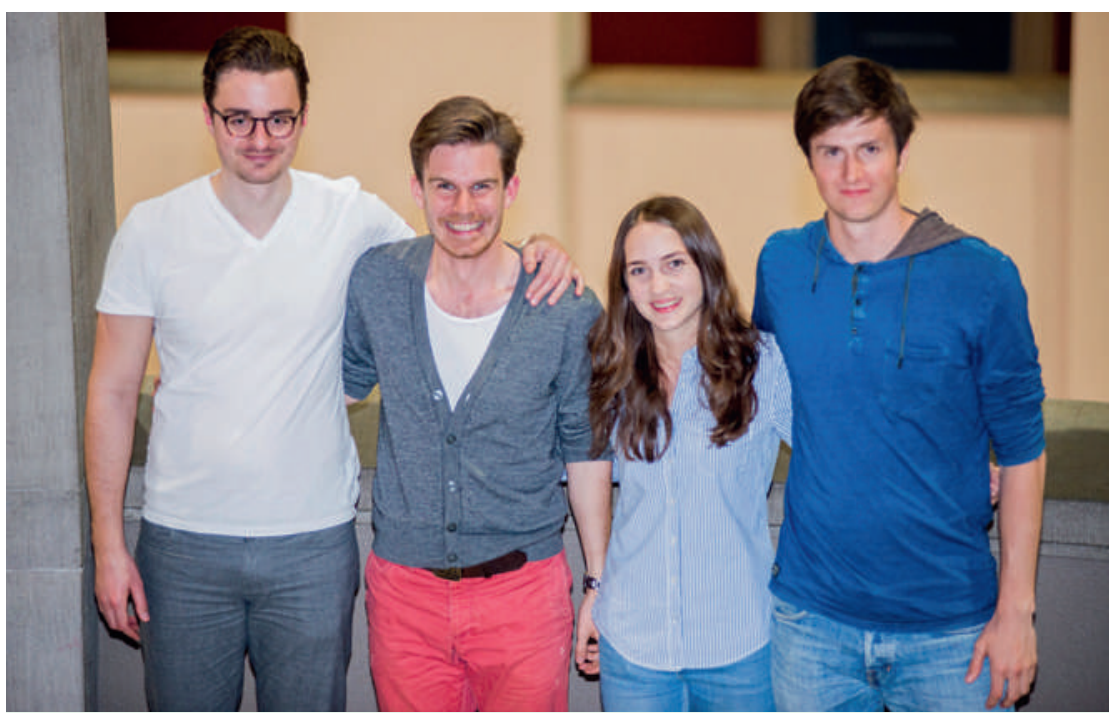

Das Organsationsteam des DocDay (v.I.n. r.): Sivert Kupfer, Niklas Stauffer, Norina Gassmann

* Das Gespräch fand Mitte August statt.
Sie organisieren Ende Oktober zum zweiten Mal den DocDay. Was ist das Ziel dieser Veranstaltung? Sivert Kupfer: Die Medizinstudierenden sollen einen Einblick erhalten in ihr zukünftiges Berufsleben. Sie können die verschiedenen Fachgesellschaften kennenlernen und sich ein Bild davon machen, welche Karrieremöglichkeiten es im Medizinberuf gibt. All das bekommen sie anderswo nicht so gut.

Es gibt sonst keine solchen Gelegenheiten während des Medizinstudiums?

Es gibt Patientenkurse in den klinischen Semestern, wo man einzelne Fachgesellschaften kennenlernen kann, und es gibt Mentoringprogramme oder den «CareerStart». Aber nur am DocDay trifft man alle Fachgesellschaften auf einmal und kann mit Unternehmungen in Kontakt treten, die Ärztinnen und Ärzte beschäftigen.

\section{und Lorenz Mürner.}

Wie gut sind die Fachgesellschaften vertreten?

Sehr gut, so wie es bis jetzt aussieht. Es sind wieder über 30 Fachgesellschaften vertreten.

Vor zwei Jahren haben Sie den DocDay zum ersten Mal durchgeführt. Was haben Sie daraus gelernt? Was ändern Sie und was machen Sie wieder gleich?

Das Grundkonzept stimmt, das haben wir beibehalten. Die Podiumsveranstaltungen und Vorträge waren jedoch eher mager besucht. Wir sind uns nicht sicher, ob es am Thema lag, oder ob diese Form den Studie- renden grundsätzlich nicht zusagt. Das Rahmenprogramm wollen wir diesmal attraktiver gestalten.

\section{Was werden Sie ändern?}

Das Programm ist noch nicht fixiert. Aber wir wollen wieder ein Podium machen, wenn möglich in $\mathrm{Zu}$ sammenarbeit mit der Ärztezeitung. Das ist zurzeit ${ }^{*}$ noch in Abklärung. Wir brauchen ein gutes Thema, denn Medizinstudierende sind eine anspruchsvolle Kundschaft.

\section{An welche Themen denken Sie?}

Ein mögliches Thema wären die Lohnunterschiede im Medizinberuf: Weshalb verdienen Angehörige bestimmter Fachrichtungen mehr als andere? Auch die Work-Life-Balance ist ein Thema, das Studierende beschäftigt. Stichworte hier sind Arbeitsmodelle oder Teilzeitarbeit.

Ihr Zielpublikum sind die Studierenden, denen Sie die Möglichkeit bieten wollen, sich umzuschauen im Hinblick auf ihre künftige Tätigkeit als Ärztinnen und Ärzte. Haben Sie sich auch zum Ziel gesetzt, das Problem des Ärztemangels anzugehen bzw. eine ausgewogenere Verteilung der Fachrichtungen zu erreichen?

Aktiv verfolgen wir dieses Ziel sicher nicht, wie denn auch. Aber passiv sorgen wir schon für einen Ausgleich: Jede Fachgesellschaft präsentiert sich am DocDay im gleichen Rahmen. Die Frage, wo man wie viel verdienen kann steht weniger im Zentrum. Man hat einen neutralen Pool von Vertreterinnen der verschiedenen Fachrichtungen, die man befragen kann.

Aber als Studierende weiss man schon, dass ein Psychiater weniger verdient als eine Radiologin?

Ja, ich denke schon.

Die Frauen bilden heute die Mehrheit der Studierenden. Verändert sich das Medizinstudium mit der Feminisierung?

Eine gute Frage. Ich denke nicht, dass es im Studium einen grossen Einfluss hat, ausser vielleicht, dass es mehr Pärchen gibt (schmunzelt). Möglich ist es schon, dass die Frauen die Männer in ihrer Denkweise beeinflussen - und umgekehrt.

Haben die Frauen einen anderen Zugang zur Medizin? Sind sie vielleicht sozial kompetenter als die Männer? Man hört ja immer, im Medizinstudium erwerbe man keine Sozialkompetenzen. 
Die Frauen sind vielleicht ein wenig einfühlsamer, aber das kann man sicherlich nicht verallgemeinern. Im Übrigen trifft es nicht mehr zu, dass die Sozialkompetenzen im Studium nicht gefördert werden. Wir lernen, dass eine Patientin mehr ist als ein Konglomerat von Organen, die in einem Buch nachgeschlagen werden können. Möglich, dass es Studierende gibt mit wenig Sozialkompetenz. Im Numerus Clausus wird das nicht selektiert.

Müsste man bei der Selektion etwas ändern?

Es ist wohl nicht ganz einfach, im Voraus festzustellen, ob jemand sozial kompetent ist oder nicht. Da gäbe es wohl eine Flut von Rekursverfahren.

Das Prestige der Ärzte sinkt - wie sehr beschäftigt Sie diese Entwicklung?

Nun, was passiert da eigentlich? Wahrscheinlich geht der Lohn nach unten. Vor allem aber glauben die Patienten nicht mehr alles, was die Ärztin oder der Arzt sagt. Aber es sind ja auch andere Berufsgruppen von einem Statusverlust betroffen, zum Beispiel die Banker. Die Bevölkerung ist generell kritischer geworden und hat nicht mehr blindes Vertrauen in die Fachleute. Vielleicht ist der Arztberuf sogar weniger als andere betroffen, weil es um die Gesundheit geht. Und die Gesundheit hat nach wie vor einen Sonderstatus.

Sie erwarten sinkende Einkommen, aber Sie investieren doch viel in Ihr Studium. Die Ausbildung dauert lange, Assistenzärztinnen arbeiten hart. Ist das noch vertretbar? Mein Eindruck ist, dass sich die Studierenden wenig Gedanken machen über wirtschaftliche und standespolitische Fragen. Die Assistenten arbeiten zudem nicht mehr so viel wie früher.

Stimmt der Eindruck, dass der hingebungsvolle Hausarzt, der rund um die Uhr für seine Patientinnen da ist, ein Auslaufmodell ist? Sind die jungen Ärztinnen nicht mehr bereit, ihr Leben dem Beruf und dem Patientenwohl zu widmen?

Das ist auch eine Generationenfrage: Ich habe gerade gestern über eine Untersuchung gelesen, wonach bei jungen Leuten die Work-Life-Balance wichtiger ist als der Lohn. Das gilt vielleicht auch für Ärzte, und es wird in Zukunft sicher weniger von ihnen geben, die rund um die Uhr verfügbar sind.

Der DocDay ist eine gesamtschweizerische Karrieremesse für Medizinstudierende. Er wird von Studierenden organisiert und findet am Samstag, 25.10.2014, an der Universität Zürich auf dem Campus Irchel statt. Ziel des DocDay ist es, angehenden Ärztinnen und Ärzten die Möglichkeit zu geben, ihre zukünftigen Berufsfelder an Messeständen zu entdecken und mit potentiellen Arbeitgebern in Kontakt zu treten. Weitere Informationen: www.docday.ch
Ist das schlimm?

Für die Patientinnen ist das sicher nicht gut, denn für die Arzt-Patienten-Beziehung ist das Vertrauen wichtig. Wenn der Arzt nur Teilzeit arbeitet, muss ein anderer einspringen, und der ganze Behandlungsprozess beginnt von vorne. Ich denke auch, dass dadurch die Gesundheitskosten steigen werden.

Immer mehr Gemeinschaftspraxen bieten geregelte Arbeitsmöglichkeiten und auch Teilzeitarbeit. Man muss nicht Hunderttausende von Franken in eine Praxis investieren ...

... und man hat fünf Wochen Ferien.

Offensichtlich ist dieses Modell erfolgreich, wenn man Praxen wie die «Permanence» im Zürcher Hauptbahnhof anschaut. Können Sie sich vorstellen, nach dem Studium so $z$ arbeiten?

Im Rahmen eines Kurses der Uni war ich in einer solchen Praxis. Für einen Arzt ist es sicherlich ein attraktiver Arbeitsort. Man muss sich weniger um die Papierarbeit kümmern. In einer Walk-in-Praxis herrscht aber auch eine unpersönlichere Atmosphäre, Gesundheitsleistungen werden wie Dienstleistungen erbracht. Wenn die Patienten dies so wollen, ist es in Ordnung. Ob eine Gemeinschaftspraxis etwas für mich als Arzt sein wird, kann ich noch nicht sagen.

Am DocDay hoffen kommerzielle Betreiber von Gemeinschaftspraxen, Arbeitskräfte rekrutieren zu können. Wollen Sie das wirklich fördern, indem Sie diese Anbieter einladen?

Wir vertreten in dieser Frage keine Meinung. Jede Firma, die ein potentieller Arbeitgeber ist, ist am DocDay willkommen. Es gibt ja die Möglichkeit, Gegensteuer zu geben, wenn andere Organisationen teilnehmen, etwa die FMH, die vor zwei Jahren noch nichts von einer Zusammenarbeit mit uns wissen wollte.

War es schwierig, Sponsoren zu finden?

Nein, eigentlich nicht. Einen Teil haben wir selber angefragt, andere sind auf uns zugekommen. Wir haben mehr Sponsoren als beim letzten Mal, so dass einige Leute denken, wir arbeiteten kommerziell, was aber nicht stimmt. Wir zahlen uns keinen Lohn aus, alle Sponsorengelder fliessen in die Veranstaltung und in den organisierenden Verein Iatroi.

Was wollen sie den Lesern der Ärztezeitung noch mitgeben? Es ist wichtig, dass den Medizinstudierenden vermehrt gezeigt wird, wie der Arztberuf aussieht und was wirtschaftlich und standespolitisch auf sie zukommt. Deshalb hoffen wir, dass möglichst viele Ärztinnen und Ärzte sich am DocDay zeigen. Die Teilnahme ist für alle offen, es gibt keine Zugangsbeschränkungen und die Teilnahme ist gratis. 